\title{
Strength of evidence for perioperative use of statins to reduce cardiovascular risk: systematic review of controlled studies
}

Anmol S Kapoor, Hussein Kanji, Jeanette Buckingham, P J Devereaux, Finlay A McAlister

\begin{abstract}
Objective To determine the strength of evidence underlying recommendations for use of statins during the perioperative period to reduce the risk of cardiovascular events.

Design Systematic review of studies with concurrent control groups.

Data sources Four electronic databases, the references of identified studies, international experts on perioperative medicine, and the authors of the primary studies.

Review methods Two reviewers independently extracted data from studies that reported acute coronary syndromes or mortality in patients receiving or not receiving statins during the perioperative period.

Main outcome measure Random effects summary odds ratios for death or acute coronary syndrome during the perioperative period.

Results 18 studies-two randomised trials $(n=177), 15$ cohort studies ( $\mathrm{n}=799$ 632), and one case-control study $(\mathrm{n}=480)$-assessed whether statins provide perioperative cardiovascular protection; 12 studies enrolled patients undergoing non-cardiac vascular surgery, four enrolled patients undergoing coronary bypass surgery, and two enrolled patients undergoing various surgical procedures. In the randomised trials the summary odds ratio for death or acute coronary syndrome during the perioperative period with statin use was $0.26(95 \%$ confidence interval 0.07 to 0.99$)$ and the summary odds ratio in the cohort studies was 0.70 (0.57 to 0.87 ). Although the pooled cohort data provided a statistically significant result, statins were not randomly allocated, results in retrospective studies were larger (odds ratio $0.65,0.50$ to 0.84 ) than those in the prospective cohorts (0.91, 0.65 to 1.27$)$, and dose, duration, and safety of statin use was not reported.

Conclusion The evidence base for routine administration of statins to reduce perioperative cardiovascular risk is inadequate.
\end{abstract}

\section{Introduction}

Each year about one million patients undergoing non-cardiac surgery worldwide and at least $10 \%$ of all patients undergoing cardiac surgery experience a cardiovascular complication. ${ }^{2}$ Some authors have endorsed the use of statins during the perioperative period to reduce the risk of cardiovascular events, ${ }^{34}$ and a recent pharmacoeconomic study stated that routine perioperative use of statins was "the most cost-effective use of statin therapy yet described."

Large randomised trials have shown that statins decrease morbidity and mortality from cardiovascular events in patients with, or at high risk of, coronary artery disease. ${ }^{6}$ Although several of the non-lipid lowering pleiotropic effects of statins (principally plaque stabilisation and anti-inflammatory modulation) are hypothesised to help prevent perioperative myocardial infarctions, the pathophysiology of perioperative myocardial infarction is incompletely understood. ${ }^{17}$

We carried out a systematic review to determine the strength of evidence for using statins during the perioperative period to reduce the risk of cardiovascular events.

\section{Methods}

We included studies if they contained data on acute coronary syndrome or mortality in adults who were or were not treated with statins during the perioperative period. We excluded studies that did not include a control group drawn from the same population, were published in abstract form only, or evaluated patients first treated with statins in the postoperative period.

\section{Study identification and selection}

On 27 September 2005 a medical librarian (JB) searched Medline (1966-2005), Embase (1988-2005), the Cochrane Library, and Biosis Previews (1969-2005) using relevant subject headings, chemical registry names, and the text words "statins" or "hydroxymethylglutaryl-coenzyme A reductase inhibitors" and "perioperative care", "preoperative care", "postoperative care" or "intra-operative care", "surgery", "surgical", and "operative/ surgical operations" (complete search terms available from JB). The search was updated on 6 February 2006. A cited reference search was also carried out in Web of Science to identify other eligible papers, reference lists of primary studies were reviewed, and experts (including primary study authors) were contacted to identify any other studies.

\section{Data extraction and quality assessment}

ASK, HK, and FAM independently screened citations, abstracted data, and assessed methodological quality. ${ }^{8}$ If data needed clarification we contacted the original investigators. As we were interested in perioperative outcomes we extracted data for 30 days after surgery (these were available for all but one of the studies after contact with the authors of the primary study-data for the final study were reported at 60 days).

\section{Data analysis}

From each study we extracted intention to treat data on death or acute coronary syndrome in the perioperative period and used RevMan 4.2 (Update Software, Oxford, United Kingdom). Owing to methodological heterogeneity among studies, we did 
not pool all studies to create an overall summary estimate, but we carried out a meta-analysis of methodologically similar studies (for example, all randomised trials or all cohort studies) using the DerSimonian and Laird random effects model to calculate odds ratios; the $\mathrm{I}^{2}$ statistic was used to assess for heterogeneity in each outcome of interest. We did not assess publication bias owing to the heterogeneity in study methodology, because covariates that lead to heterogeneity in the relation between exposure and outcome will also lead to asymmetry in a funnel plot. ${ }^{10}$

\section{Results}

Overall, 2373 citations were identified, of which 18 studies fulfilled our eligibility criteria (fig 1), ${ }^{\mathrm{w} 1 \mathrm{w} 18}$ including eight studies not included in a recently published review on the potential of statins used during the perioperative period. ${ }^{3}$

Of the studies eligible for inclusion, three were reported in more than one publication. The second publication reported results for different end points, different follow-up periods, or selected subgroups. ${ }^{11-13}$ w4 w13 w14 Some overlap also occurred in patient populations reported in a case-control publication ${ }^{\mathrm{w} 6}$ and in a retrospective cohort publication. ${ }^{\mathrm{w} 13}$ On the advice of the author of the primary study we included the data from only the retrospective cohort publication in any pooled estimates to avoid potential double counting of some patients (D Poldermans, personal communication, 2006).

Table 1 presents key characteristics from the 18 unique included studies, reporting data from 800289 operations in 800106 patients. Two were randomised controlled trials ${ }^{\mathrm{w} 1 \mathrm{w1} 4}$; one was a case-control study ${ }^{\mathrm{w} 6}$; and 15 were cohort studies, of which 12 used a retrospective design. ${ }^{12-w 5}$ w7-w13 w15-w18 One of these cohort studies reported data derived from a randomised trial testing ex vivo application of edifoligide to vein grafts before implantation. ${ }^{\mathrm{w} 16}$ All but two of the included studies $^{\mathrm{w} 7}{ }^{\mathrm{w} 18}$ evaluated outcomes with statin use in patients undergoing cardiovascular surgery (four studies of coronary artery bypass grafting surgery and 12 of non-cardiac vascular surgical procedures). Although two studies examined the effects of lipid lowering therapy rather than statin therapy, in both ${ }^{\mathrm{w} 7}$ w17 the majority of patients receiving lipid lowering therapy were using statins, and event rates in statin users and non-users were identical to those of users of other lipid lowering therapy ( $\mathrm{G}$ Landesberg, personal communication, 2006).

\section{Qualitative data synthesis}

The Jadad quality scores, out of a maximum of five, for the two randomised trials were five and two, but in one of the trials it was only clear that treatment allocation had been adequately concealed after contact with the author. ${ }^{\mathrm{w} 1}$

Overall the 16 non-randomised studies were rated as being of good quality using the Downs and Black scoring system (table 2). ${ }^{8}$ The internal validity scores for the 16 non-randomised studies were, however, only fair to moderate, with lack of blinding of patients or providers to statin exposure being the main shortfall. Few of these studies described the duration or dose of statin therapy during the perioperative period and few reported information on compliance with treatment.

\section{Perioperative death or acute coronary syndrome}

Both of the randomised trials and 13 of the cohort studies evaluated the composite outcome of perioperative death or acute coronary syndrome. Although the summary odds ratio for the randomised trials was 0.26 (95\% confidence interval 0.07 to 0.99 ), this was based on only 13 events in 177 patients and cannot be considered conclusive. However, pooling the data from all 13 cohort studies (1004 events in 18463 patients) gave a summary odds ratio of 0.70 (0.57 to 0.87 ) with statin use (fig 2).

Although event rates in controls were similar for the cohorts of non-cardiac surgery $(6.3 \%)$ and cardiac surgery studies $(6.6 \%)$, heterogeneity was present in the pooled estimate derived from these 13 cohort studies, largely driven by the lower event rates in statin treated patients in four of the smallest studies, all of which reported fewer than 50 events. ${ }^{\text {w3 }}$ w12 w13 w18 The pooled estimate from the 10 retrospective cohort studies (odds ratio $0.65,95 \%$ confidence interval 0.50 to 0.84 ) suggested substantially greater benefits with statin use than that derived from the three prospective cohort studies (0.91, 0.65 to 1.27$)$.

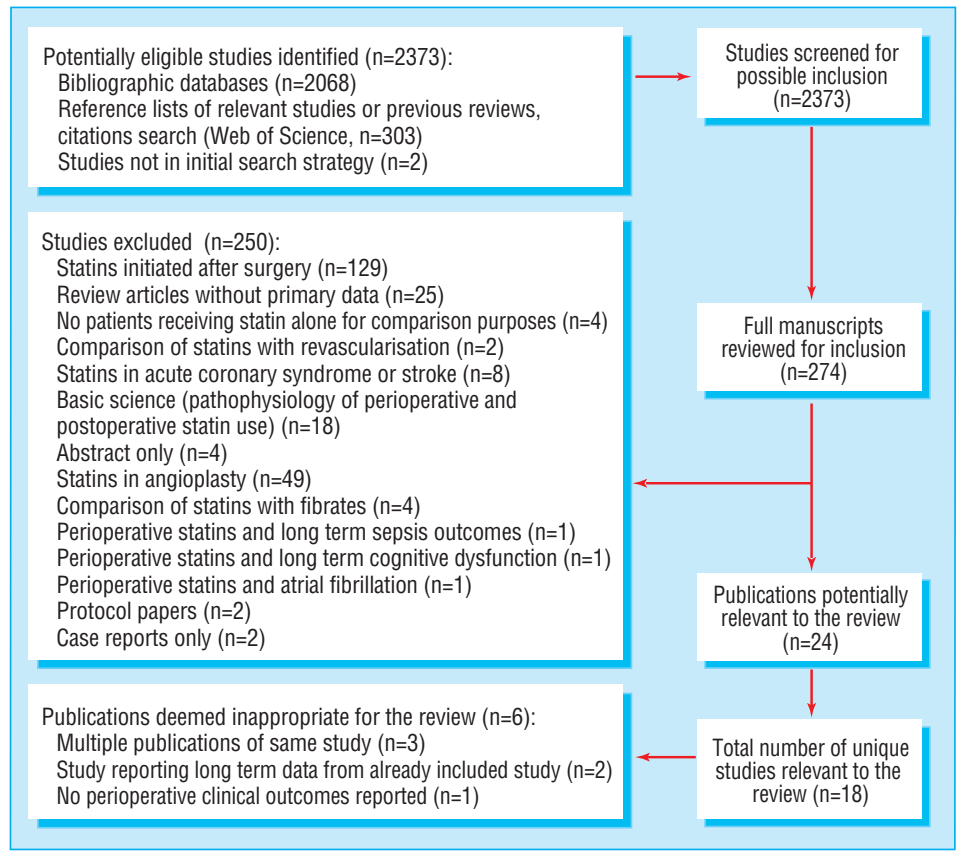

Fig 1 Flow of studies through selection process 
Research

Table 1 Description of included studies

\begin{tabular}{|c|c|c|c|c|c|c|c|}
\hline Study (design) & $\begin{array}{l}\text { Sample } \\
\text { size (mean } \\
\text { age) }\end{array}$ & $\begin{array}{c}\text { Study population and types of } \\
\text { surgery }\end{array}$ & Statin use & $\begin{array}{c}\text { Patients with } \\
\text { dyslipidaemia (\%) }\end{array}$ & $\begin{array}{c}\text { Patients using aspirin } \\
\text { or other antiplatelet } \\
\text { agent, or } \beta \text { blockers } \\
(\%)\end{array}$ & Outcomes & Follow-up \\
\hline \multicolumn{8}{|l|}{ Non-cardiac surgery: } \\
\hline $\begin{array}{l}\text { Boersma et al } 2001^{w 15} \\
\text { (prospective cohort) }\end{array}$ & 1351 (NR) & $\begin{array}{l}\text { Consecutive patients } \\
\text { scheduled for elective major } \\
\text { vascular surgery ( } 47 \% \text { aortic } \\
\text { femoral bypass, } 35 \% \\
\text { infrainguinal reconstruction) }\end{array}$ & $\begin{array}{l}\text { Taking "lipid } \\
\text { lowering } \\
\text { agents" before } \\
\text { operation }\end{array}$ & NR & 30,27 & $\begin{array}{l}\text { Cardiac death or non-fatal } \\
\text { acute coronary syndrome } \\
\text { detected by routine clinical } \\
\text { care }^{\star}\end{array}$ & 1 month \\
\hline $\begin{array}{l}\text { Poldermans et al } 2003^{\mathrm{w} 6} \\
\text { (case control) }\end{array}$ & $480(70)$ & $\begin{array}{l}\text { Patients undergoing major } \\
\text { vascular surgery ( } 38 \% \text { repair } \\
\text { of acute abdominal aortic } \\
\text { aneurysm, } 48 \% \text { elective repair } \\
\text { of abdominal aortic aneurysm, } \\
11 \% \text { revascularisation of legs) } \\
\text { from } 1991-2000\end{array}$ & $\begin{array}{l}\text { Taking statins } \\
\text { before } \\
\text { operation }\end{array}$ & 45 & 26,30 & $\begin{array}{l}\text { Death from any cause within } \\
30 \text { days of surgery }\end{array}$ & 1 month \\
\hline $\begin{array}{l}\text { Landesberg et al 2003 } \\
\text { (retrospective cohort) }\end{array}$ & $502(66)$ & $\begin{array}{l}\text { Consecutive patients } \\
\text { undergoing elective vascular } \\
\text { operations ( } 60 \% \text { infrainguinal } \\
\text { bypass, } 38 \% \text { repair of } \\
\text { abdominal aortic aneurysm, } \\
2 \% \text { axillofemoral bypass) from } \\
1990-9\end{array}$ & $\begin{array}{l}\text { Taking lipid } \\
\text { lowering } \\
\text { therapy before } \\
\text { operation }\end{array}$ & 21 & NR, 15 & Death from any cause & $\begin{array}{l}1 \text { month and } \\
\text { long term }\end{array}$ \\
\hline $\begin{array}{l}\text { Durazzo et al } \mathrm{al}^{\mathrm{w} 1} 2004 \\
\text { (randomised controlled } \\
\text { trial) }\end{array}$ & 100 (NR) & $\begin{array}{l}\text { Patients for elective } \\
\text { non-cardiac vascular surgery } \\
(56 \% \text { aortic repair, } 20 \% \\
\text { infrainguinal arterial bypass, } \\
11 \% \text { carotid endarterectomy), } \\
\text { no recent acute coronary } \\
\text { syndrome or stroke, recruited } \\
\text { between } 1999 \text { and } 2000\end{array}$ & $\begin{array}{l}\text { Taking statins } \\
\text { for at least } 14 \\
\text { days before } \\
\text { operation }\end{array}$ & 63 & 39,60 & $\begin{array}{l}\text { Cardiac death; non-fatal acute } \\
\text { coronary syndromes or } \\
\text { ischaemic stroke, or both } \\
\text { detected by routine physical } \\
\text { examinations, } \\
\text { electrocardiography, and } \\
\text { cardiac biomarkers done } \\
\text { postoperatively for seven } \\
\text { days. After discharge, only } \\
\text { symptomatic events detected† }\end{array}$ & $\begin{array}{l}10 \text { days and } 6 \\
\text { months }\end{array}$ \\
\hline $\begin{array}{l}\text { Lindenauer et al }{ }^{\mathrm{w7}} 2004 \\
\text { (retrospective cohort) }\end{array}$ & $\begin{array}{c}780591 \\
(64)\end{array}$ & $\begin{array}{l}\text { Patients who survived beyond } \\
\text { postoperative day } 2 \text { after } \\
\text { non-cardiac surgery ( } 50 \% \text { of } \\
\text { surgery elective and types of } \\
\text { surgery: } 36 \% \text { orthopaedic, } \\
32 \% \text { abdominal, } 8 \% \text { vascular) } \\
\text { from } 2000-1\end{array}$ & $\begin{array}{l}\text { Taking lipid } \\
\text { lowering } \\
\text { therapy in first } \\
\text { two days after } \\
\text { admission to } \\
\text { hospital ( } 91 \% \\
\text { statins) }\end{array}$ & 5 & 11,19 & $\begin{array}{l}\text { In-hospital mortality (average } \\
\text { stay five days) }\end{array}$ & Admission to hospital \\
\hline $\begin{array}{l}\text { Kertai et al 2004 } \\
\text { (retrospective cohort) }\end{array}$ & 570 (68) & $\begin{array}{l}\text { Patients undergoing elective } \\
\text { surgery for infrarenal } \\
\text { abdominal aortic aneurysm } \\
\text { between } 1991 \text { and } 2000\end{array}$ & $\begin{array}{l}\text { Taking statins } \\
\text { before } \\
\text { operation }\end{array}$ & NR & 23,45 & $\begin{array}{l}\text { All cause mortality and } \\
\text { myocardial infarction detected } \\
\text { by routine electrocardiography } \\
\text { and cardiac biomarkers done } \\
\text { postoperatively for seven } \\
\text { days. After discharge, only } \\
\text { symptomatic events detected } †\end{array}$ & 1 month \\
\hline $\begin{array}{l}\text { Abbruzzese et al 2004 } \\
\text { (retrospective cohort) }\end{array}$ & $\begin{array}{c}189 \\
\text { operations, } \\
172 \text { patients } \\
(69)\end{array}$ & $\begin{array}{l}\text { Consecutive patients who } \\
\text { underwent infrainguinal } \\
\text { arterial reconstruction from } \\
1999-2001\end{array}$ & $\begin{array}{l}\text { Taking statins } \\
\text { before } \\
\text { operation }\end{array}$ & NR & $63, N R$ & $\begin{array}{l}\text { All cause mortality and } \\
\text { myocardial infarction extracted } \\
\text { from chart review }{ }^{\star}\end{array}$ & 1 month \\
\hline $\begin{array}{l}\text { 0'Neil-Callahan et al } \\
2005^{2} \text { (retrospective } \\
\text { cohort) }\end{array}$ & $\begin{array}{c}1163 \\
\text { operations, } \\
997 \text { patients } \\
(71)\end{array}$ & $\begin{array}{l}\text { Consecutive patients who } \\
\text { underwent carotid } \\
\text { endarterectomy ( } 31 \%) \text {, aortic } \\
\text { surgery }(15 \%) \text { or } \\
\text { revascularisation of the legs } \\
(54 \%) \text { from } 1999-2000(84 \% \\
\text { elective) }\end{array}$ & $\begin{array}{l}\text { Taking statins } \\
\text { before } \\
\text { operation }\end{array}$ & 52 & 52,49 & $\begin{array}{l}\text { Death and acute coronary } \\
\text { syndrome extracted from } \\
\text { chart review }^{\star}\end{array}$ & Admission to hospital \\
\hline $\begin{array}{l}\text { Schouten et al 2005 } \\
\text { (retrospective cohort) }\end{array}$ & 981 (67) & $\begin{array}{l}\text { Consecutive patients } \\
\text { undergoing elective major } \\
\text { vascular surgery from } \\
1998-2004 \text { (60\% repair of } \\
\text { abdominal aortic aneurysm, } \\
40 \% \text { revascularisation of the } \\
\text { legs) }\end{array}$ & $\begin{array}{l}\text { Taking statins } \\
\text { before } \\
\text { operation } \\
\text { (average } 40 \\
\text { days) }\end{array}$ & NR & NR, NR & $\begin{array}{l}\text { Myopathy, death, or acute } \\
\text { coronary syndrome detected } \\
\text { by routine electrocardiography } \\
\text { and cardiac biomarkers done } \\
\text { postoperatively for seven days }\end{array}$ & 7 days \\
\hline $\begin{array}{l}\text { Kennedy et al } 2005^{\mathrm{w} 5} \\
\text { (retrospective cohort) }\end{array}$ & $3283(72)$ & $\begin{array}{l}\text { Consecutive patients } \\
\text { undergoing carotid } \\
\text { endarterectomy ( } 62 \% \\
\text { symptomatic) from } 2000-1\end{array}$ & $\begin{array}{l}\text { Taking statins } \\
\text { before } \\
\text { operation }\end{array}$ & NR & $N R, N R$ & $\begin{array}{l}\text { Mortality, stroke, or acute } \\
\text { coronary syndromes extracted } \\
\text { from chart review }{ }^{*}\end{array}$ & Admission to hospital \\
\hline $\begin{array}{l}\text { McGirt et al 2005 } \\
\text { (retrospective cohort) }\end{array}$ & $1566(72)$ & $\begin{array}{l}\text { Consecutive patients } \\
\text { undergoing carotid } \\
\text { endarterectomy ( } 42 \% \\
\text { symptomatic) from } 1994-2004\end{array}$ & $\begin{array}{l}\text { Taking statins } \\
\text { for at least } \\
\text { seven days } \\
\text { before } \\
\text { operation }\end{array}$ & 50 & NR, NR & $\begin{array}{l}\text { Mortality, stroke, or acute } \\
\text { coronary syndromes extracted } \\
\text { from chart review }{ }^{\star}\end{array}$ & 1 month \\
\hline $\begin{array}{l}\text { Ward et al 2005 } \\
\text { (retrospective cohort) }\end{array}$ & $446(67)$ & $\begin{array}{l}\text { Consecutive patients } \\
\text { undergoing infrainguinal } \\
\text { vascular bypass surgery from } \\
\text { 1995-2001 because of } \\
\text { atherosclerotic disease }\end{array}$ & $\begin{array}{l}\text { Taking statins } \\
\text { before } \\
\text { operation }\end{array}$ & NR & NR, 21 & $\begin{array}{l}\text { Mortality, stroke, acute } \\
\text { coronary syndromes, or major } \\
\text { vascular complications } \\
\text { extracted from chart review* }\end{array}$ & 1 month \\
\hline
\end{tabular}




\begin{tabular}{|c|c|c|c|c|c|c|c|}
\hline Study (design) & $\begin{array}{l}\text { Sample size } \\
\text { (mean age) }\end{array}$ & $\begin{array}{c}\text { Study population and types of } \\
\text { surgery }\end{array}$ & Statin use & $\begin{array}{c}\text { Patients with } \\
\text { dyslipidaemia (\%) }\end{array}$ & $\begin{array}{l}\text { Patients using aspirin or } \\
\text { other antiplatelet agent, } \\
\text { or } \beta \text { blockers (\%) }\end{array}$ & Outcomes & Follow-up \\
\hline $\begin{array}{l}\text { Amar et al } 2005^{w 18} \\
\text { (prospective cohort) }\end{array}$ & 131 (71) & $\begin{array}{l}\text { Patients aged } 60 \text { years or } \\
\text { older undergoing elective } \\
\text { major thoracic surgery from } \\
2002-3 \text { (89\% lobectomy, } 7 \% \\
\text { oesophagectomy, } 4 \% \\
\text { pneumonectomy) }\end{array}$ & $\begin{array}{l}\text { Taking statins } \\
\text { before } \\
\text { operation }\end{array}$ & NR & NR, 22 & $\begin{array}{l}\text { Death within } 30 \text { days, atrial } \\
\text { fibrillation, stroke extracted } \\
\text { from hospital and physician } \\
\text { records }\end{array}$ & 1 month \\
\hline $\begin{array}{l}\text { Conte et al } 2005^{\text {w16 }} \\
\text { (prospective cohort in } \\
\text { setting of randomised } \\
\text { controlled trial) }\end{array}$ & 1404 (69) & $\begin{array}{l}\text { Patients undergoing } \\
\text { infrainguinal revascularisation } \\
\text { for critical limb ischaemia and } \\
\text { enrolled in a randomised trial } \\
\text { testing ex vivo application of } \\
\text { edifoligide to vein grafts } \\
\text { before implantation }\end{array}$ & $\begin{array}{l}\text { Taking statins } \\
\text { before } \\
\text { operation }\end{array}$ & 55 & 50,49 & $\begin{array}{l}\text { Mortality, acute coronary } \\
\text { syndromes, and stroke } \\
\text { collected prospectively within } \\
\text { trial setting }\end{array}$ & 1 month \\
\hline \multicolumn{8}{|l|}{ Cardiac surgery: } \\
\hline $\begin{array}{l}\text { Christenson 1999'14 } \\
\text { (randomised controlled } \\
\text { trial) }\end{array}$ & 77 (63) & $\begin{array}{l}\text { Consecutive patients } \\
\text { undergoing coronary artery } \\
\text { bypass grafting surgery ( } 87 \% \\
\text { elective) between } 1997 \text { and } \\
1998\end{array}$ & $\begin{array}{l}\text { Taking statins } \\
\text { for four weeks } \\
\text { before } \\
\text { operation }\end{array}$ & 62 & $N R, N R$ & $\begin{array}{l}\text { Acute coronary syndrome } \\
\text { detected by routine } \\
\text { electrocardiography and } \\
\text { cardiac biomarkers done } \\
\text { postoperatively for seven } \\
\text { days. After discharge, only } \\
\text { symptomatic events detected } \dagger\end{array}$ & $\begin{array}{l}7 \text { days and } 12 \\
\text { months }\end{array}$ \\
\hline $\begin{array}{l}\text { Dotani et al } 2000^{w 12} \\
\text { (retrospective cohort) }\end{array}$ & $323(63)$ & $\begin{array}{l}\text { Consecutive elective patients } \\
\text { undergoing coronary artery } \\
\text { bypass grafting surgery in } \\
1997\end{array}$ & $\begin{array}{l}\text { Taking statins } \\
\text { before } \\
\text { operation }\end{array}$ & 50 & NR, NR & $\begin{array}{l}\text { Cardiac death, acute coronary } \\
\text { syndromes, stroke, } \\
\text { arrhythmia, or heart failure } \\
\text { extracted from chart review* }\end{array}$ & $\begin{array}{l}2 \text { months and } \\
12 \text { months }\end{array}$ \\
\hline $\begin{array}{l}\text { Pan et al } 2004^{\mathrm{w} 11} \\
\text { (retrospective cohort) }\end{array}$ & $1663(63)$ & $\begin{array}{l}\text { Consecutive patients } \\
\text { undergoing coronary artery } \\
\text { bypass grafting surgery ( } 81 \% \\
\text { elective) between } 2000 \text { and } \\
2001\end{array}$ & $\begin{array}{l}\text { Taking statins } \\
\text { before } \\
\text { operation }\end{array}$ & 66 & 51,52 & $\begin{array}{l}\text { All cause mortality, acute } \\
\text { coronary syndrome, stroke, } \\
\text { arrhythmias, or renal } \\
\text { dysfunction extracted from } \\
\text { chart review* }^{\star}\end{array}$ & 1 month \\
\hline $\begin{array}{l}\text { Ali and Buth 2005w4 } \\
\text { (retrospective cohort) }\end{array}$ & 5469 (NR) & $\begin{array}{l}\text { Consecutive patients } \\
\text { undergoing coronary artery } \\
\text { bypass grafting surgery or } \\
\text { valve surgery, or both }(51 \% \\
\text { elective) from 1998-2003 }\end{array}$ & $\begin{array}{l}\text { Taking statins } \\
\text { before } \\
\text { operation }\end{array}$ & NR & NR, 81 & $\begin{array}{l}\text { Mortality, intra-aortic balloon } \\
\text { pump use, or acute coronary } \\
\text { syndrome (extracted from } \\
\text { discharge records)* }\end{array}$ & 7 days \\
\hline
\end{tabular}

NR=not reported.

*Thus clinically apparent cases only.

†That is, no routine surveillance.

The data we report in figure 2 are derived from the crude event rates reported in each study. Those studies that examined for differences between statin users and non-users reported that statin users were more likely to be male and to have coronary artery disease or other cardiovascular comorbidities. ${ }^{\mathrm{w} 2} \mathrm{w} 3 \mathrm{w}$ (57 $\mathrm{w} 10 \mathrm{w13}$ As a result, those studies that adjusted for covariates found that the associations between statin use and outcomes were preserved

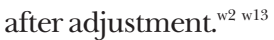

\section{Perioperative death and safety}

Although neither of the randomised trials were powered for mortality (only one death occurred in the perioperative period in the 177 patients randomised), the pooled odds ratios from the cohort studies were 0.49 ( 0.38 to 0.64$)$ for cardiac surgery studies and 0.69 (0.65 to 0.72 ) for non-cardiac surgery studies (fig 3). Again, those studies that adjusted for covariates found that the associations between statin use and outcomes were still present

Table 2 Methodological quality assessments of non-randomised studies. Values in brackets are maximum scores achievable

\begin{tabular}{|c|c|c|c|c|c|c|}
\hline \multirow[b]{2}{*}{ Study (design) } & \multicolumn{6}{|c|}{ Downs and Black quality score (maximum number) ${ }^{8}$} \\
\hline & Reporting (11) & $\begin{array}{c}\text { External validity } \\
\text { (3) }\end{array}$ & $\begin{array}{l}\text { Internal validity: } \\
\text { bias (7) }\end{array}$ & $\begin{array}{l}\text { Internal validity: } \\
\text { confounding (6) }\end{array}$ & Power (2) & Overall (29) \\
\hline Boersma et al $2001^{\text {w15 }}$ (prospective cohort) & 10 & 3 & 4 & 4 & 2 & 23 \\
\hline Poldermans et al $2003^{\mathrm{w} 6}$ (case-control) & 10 & 2 & 4 & 4 & 2 & 22 \\
\hline Landesberg et al $2003^{\text {w17 }}$ (retrospective cohort) & 7 & 3 & 4 & 4 & 1 & 19 \\
\hline Lindenauer et al 2004 ${ }^{\mathrm{w} 7}$ (retrospective cohort) & 10 & 2 & 4 & 4 & 2 & 22 \\
\hline Kertai et al $2004^{w 13}$ (retrospective cohort) & 9 & 3 & 4 & 3 & 2 & 21 \\
\hline Abbruzzese et al 2004 $4^{\text {w10 }}$ (retrospective cohort) & 9 & 3 & 4 & 3 & 2 & 22 \\
\hline O'Neil-Callahan et al $2005^{\mathrm{w} 2}$ (retrospective cohort) & 8 & 3 & 5 & 4 & 2 & 21 \\
\hline Schouten et al $2005^{\text {w8 }}$ (retrospective cohort) & 9 & 3 & 4 & 3 & 2 & 21 \\
\hline Kennedy et al $2005^{w 5}$ (retrospective cohort) & 10 & 3 & 3 & 4 & 2 & 22 \\
\hline McGirt et al $2005^{\mathrm{w} 3}$ (retrospective cohort) & 11 & 3 & 4 & 4 & 2 & 24 \\
\hline Ward et al $2005^{\mathrm{w} 9}$ (retrospective cohort) & 9 & 3 & 4 & 4 & 2 & 22 \\
\hline Amar et al $2005^{w 18}$ (prospective cohort) & 6 & 2 & 3 & 3 & 1 & 15 \\
\hline Conte et al $2005^{\text {w16 }}$ (prospective cohort) & 8 & 1 & 3 & 4 & 1 & 17 \\
\hline Dotani et al $2000^{\mathrm{w} 12}$ (retrospective cohort) & 9 & 3 & 4 & 3 & 2 & 22 \\
\hline Pan et al 2004 $4^{\mathrm{w} 11}$ (retrospective cohort) & 8 & 3 & 3 & 4 & 2 & 20 \\
\hline Ali and Buth $2005^{\mathrm{w} 4}$ (retrospective cohort) & 10 & 3 & 3 & 4 & 2 & 22 \\
\hline
\end{tabular}


even after adjustment. ${ }^{\mathrm{w} 7}$ w11 Further, a case-control study with rigorous matching of controls to cases reported an odds ratio for mortality with statin use of $0.22(0.10$ to 0.47$)$ and consistency of effect across all tested subgroups. ${ }^{\mathrm{w}}{ }^{\mathrm{w}}$

Only one of the studies reported liver dysfunction rates in statin users (one case of elevated aminotransferase levels in 50 patients) ${ }^{\mathrm{w} 1}$ and the only study that explicitly examined risk of rhabdomyolysis in surgical patients who did or did not take statins reported no increased risk with statin use; however, the frequency of increased creatinine phosphokinase levels to greater than 10 times normal in both arms of that study $(8 \% v$

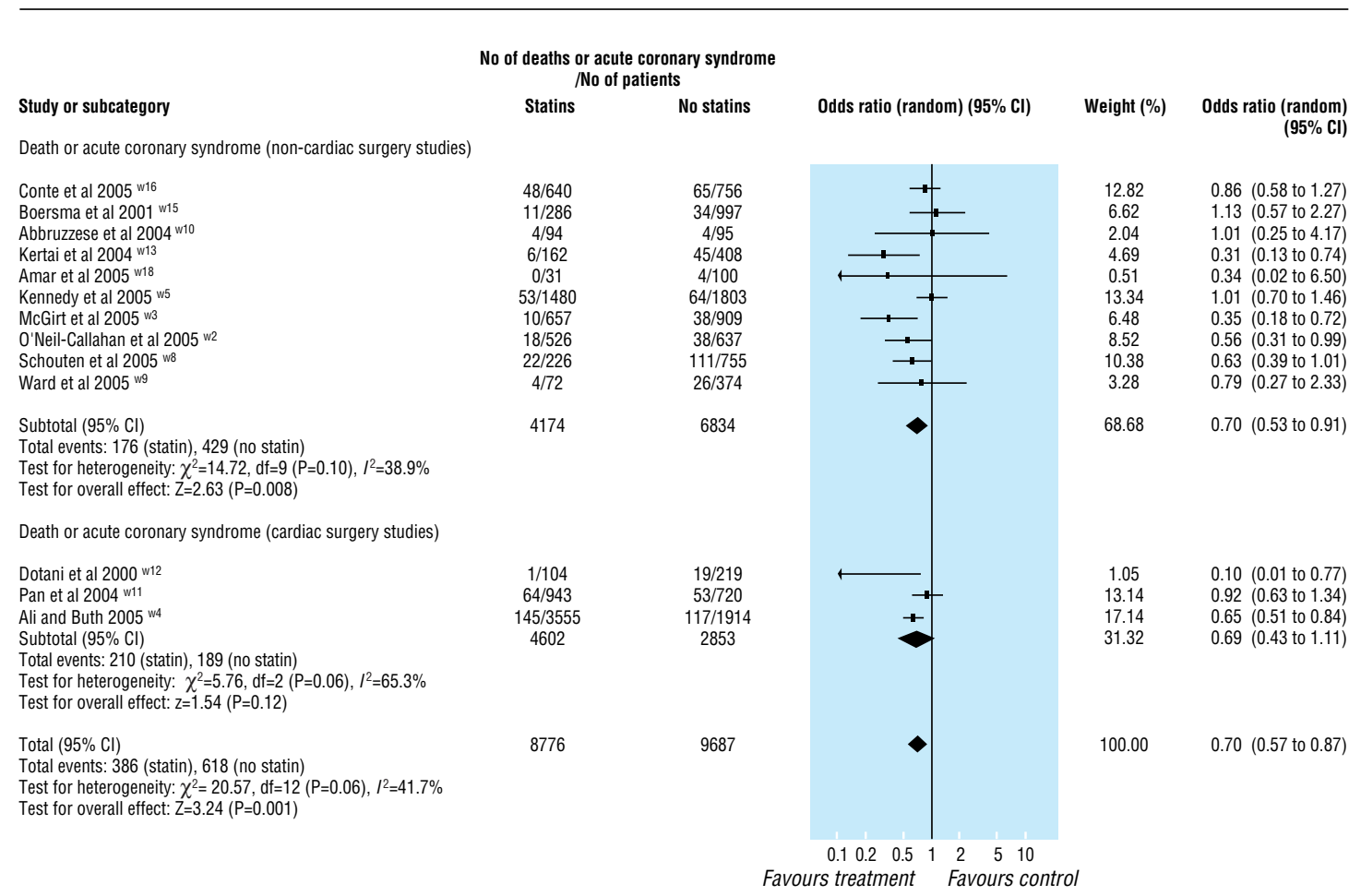

Fig 2 Perioperative death or acute coronary syndrome event rates in cohort studies

\section{Study or subcategory}

Death (non-cardiac surgery studies)

Conte et al 2005 w16

Landesberg et al $2003^{\text {w17 }}$

Abbruzzese et al 2004 w10

Lindenauer et al $2004 \mathrm{w} /$

Amar et al 2005 w18

Kennedy et 2005

Kennedy et al 2005

O'Neil-Callahan ot al 2005 w2

Schouten et a 2005 w8

Ward et al 2005 w9

Subtotal $(95 \% \mathrm{Cl})$

Total events: 1677 (statin), 21581 (no statin)

Test for heterogeneity: $\chi^{2}=8.89, \mathrm{df}=9(\mathrm{P}=0.45), I^{2}=0 \%$

Test for overall effect: $Z=14.54(P<0.0001)$

Death (cardiac surgery studies)

Dotani et al 2000 w12
Pan et al 2004 w11
Ali and Buth 2005 w4
Subtotal $(95 \%$ Cl)
Total events: 109 (statin), 131 (no statin)
Test for heterogeneity: $\chi^{2}=1.00$, df $=2(P=0.61), I^{2}=0 \%$
Test for overall effect: $Z=5.31$ ( $P<0.0001$ )

Test for overall effect: $Z=5.31(P<0.0001)$

Total $(95 \% \mathrm{Cl})$

Total events: 1786 (statin), 21712 (no statin)

Test for heterogeneity: $\chi^{2}=16.01, d f=12(\mathrm{P}=0.19), l^{2}=25.1 \%$

Test for overall effect: $Z=5.13(P=0.0001)$

Fig 3 Perioperative death rates in cohort studies

\section{No of deaths/No of patients}

Statins

No statins

$\begin{array}{cc}12 / 640 & 26 / 756 \\ 1 / 62 & 6 / 440 \\ 2 / 94 & 3 / 95 \\ 1640 / 77082 & 21460 / 703509 \\ 0 / 31 & 2 / 100 \\ 7 / 1480 & 21 / 1803 \\ 2 / 657 & 19 / 909 \\ 6 / 526 & 5 / 367 \\ 5 / 226 & 30 / 755 \\ 2 / 72 & 9 / 374 \\ & \end{array}$

80870

709378

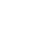

$\begin{array}{cc}0 / 104 & 8 / 219 \\ 17 / 943 & 27 / 720 \\ 92 / 3555 & 96 / 1914 \\ 4602 & 2853 \\ & \\ & \\ 85472 & 712231\end{array}$

712231

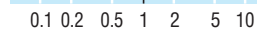

Favours treatment Favours control 
$10 \%)^{\text {w8 }}$ was substantially higher than the rates reported in the medical trials of statins. ${ }^{6}$

\section{Implications for trial design}

Assuming that the event rates in the statin studies we analysed are representative of what can be expected in patients undergoing vascular surgery, if the composite of perioperative death or acute coronary syndrome was the primary outcome then 3000 patients per arm would be required for a placebo controlled randomised trial to establish whether statins reduced this outcome by $25 \%$; if the primary outcome was perioperative death then over 7000 patients would be required per arm for a placebo controlled randomised trial to establish whether statins reduced that outcome.

Data on the effect of perioperative statins on liver and muscle function currently are limited in the literature. It is not inconceivable, however, that perioperative statins may create a higher risk for these events than statins used in routine medical practice because patients requiring vascular surgery tend to be older and often have comorbidities that would have excluded them from statin trials. Thus it is important that any randomised trials evaluating perioperative statin use include assessments for these safety outcomes.

\section{Discussion}

Literature suggests that the use of statins during the perioperative period in patients undergoing high risk surgery may confer substantial benefits. Statin users exhibit perioperative rates of death or acute coronary syndromes that are $30 \%$ to $42 \%$ lower than those observed in patients who are not taking statins at the time of surgery. It is important to emphasise that these findings are largely based on observational cohort studies and that the two randomised trials published on this topic are too small (even when pooled) to provide conclusive evidence on the effect of statins in the perioperative period.

The perioperative benefits of statins reported in the literature are greater than the benefits reported for long term statin use in patients with coronary disease ${ }^{6}$ or after coronary artery bypass grafting surgery ${ }^{14}$; the magnitude of risk reduction seen in these perioperative studies even exceeds the benefits seen with statin use in the immediate period after a myocardial infarction. ${ }^{15} 16$ Although some authors have speculated that the non-lipid lowering pleiotropic effects of statins may be particularly beneficial during the postoperative period in patients in whom levels of inflammatory cytokines and chemokines are at their highest, ${ }^{17}$ an equally appealing pathophysiological rationale was cited by experts who endorsed hormone replacement therapy in the 1990s (on the basis of stronger observational evidence than that currently available for perioperative statins). ${ }^{18}$ Even with adjustment for covariates and prescription propensity, the use of statins in an observational dataset may just be a surrogate for unmeasured confounders that improve prognosis: for example, the use of other therapies proved to be efficacious in cardiovascular risk reduction such as aspirin, $\beta$ blockers, and angiotensin converting enzyme inhibitors may be more common in statin users. Our study summarises the current literature on this topic but should not be taken as an endorsement for the widespread use of statins perioperatively. Rather, our study should be used to inform the design of a randomised trial of statins in the perioperative period.

As with any systematic review, our study has limitations beyond the paucity of evidence for statins from randomised trials. For example, none of the studies reported doses, few reported the duration of therapy before surgery, and none pro- vided details on patient compliance with treatment. One of the key criteria for establishing causation between an exposure and an outcome is to show a dose-response relation; we were unable to do this given the status of the current literature. Furthermore, few studies reported the type of statins used, so that it was not possible to examine for differences between agents. Also, data were limited on cholesterol levels before and after surgery in virtually all of these studies; it was therefore not possible to examine statin effects according to baseline lipid levels. We cannot establish to what extent the apparent benefits seen with statins in these observational studies were inflated owing to the inclusion of patients withdrawn from their statin before surgery in the group not using statins (a potential confounder since acute statin withdrawal may cause cardiac events). ${ }^{19}{ }^{20}$ Finally, although our review included data from over 800000 patients, these studies provided little information on the safety of perioperative statin use.

In conclusion, although our systematic review suggests substantial benefits from perioperative statin use, this is largely based on observational data, and evidence from the randomised trials is not conclusive even when pooled. We believe it is reasonable to advocate that statins should be started preoperatively in eligible patients who would warrant statin therapy for medical reasons independent of the proposed operation-for example, patients with coronary disease, patients with multiple cardiac risk factors, or patients with elevated low density lipoprotein cholesterol levels. However, until evidence from randomised trials has accumulated sufficient numbers of perioperative events to provide a definitive answer, we believe it is premature to advocate the routine use of statins in the perioperative period for patients without established coronary disease.

We thank J Kennedy, D Poldermans, MS Conte, G Landesberg, and D Amar for providing additional unpublished information about their studies.

Contributors: ASK and HK contributed equally to the study. ASK, HK, and FAM conceived and designed the study.JB carried out the literature search. ASK, HK, and FAM reviewed the literature search and extracted all study data. FAM wrote the first draft of the paper and all authors contributed to subsequent drafts. FAM is guarantor.

Funding: This project was unfunded; FAM is supported by a health scholar award from the Alberta Heritage Foundation for Medical Research, a new investigator award from the Canadian Institutes of Health Research, and the Merck Frosst/Aventis chair in patient health management at the University of Alberta. PJD is supported by a new investigator award from the Canadian Institutes of Health Research.

Competing interests: None declared.

Ethical approval: Not required.

1 Devereaux PJ, Goldman L, Cook DJ, Gilbert K, Leslie K, Guyatt GH. Perioperative cardiac events in patients undergoing noncardiac surgery: a review of the magnitude of

\section{What is already known on this topic}

Several authors advocate using statins to reduce perioperative cardiovascular risk

The evidence for such use is unclear

\section{What this study adds}

Perioperative statins are associated with lower rates of acute coronary syndromes and mortality

These findings are based on 16 observational studies and only two small randomised trials

Evidence is insufficient to advocate the routine use of statins for perioperative cardiovascular risk reduction 
the problem, the pathophysiology of the events and methods to estimate and communicate risk. CMAJ 2005;173:627-34

2 Mangano DT. Cardiovascular morbidity and CABG surgery-a perspective: epidemiology, cost and potential therapeutic solution.J Cardiovasc Surg 1995;10:366-8.

3 Biccard BM, Sear JW, Foex P. Statin therapy: a potentially useful peri-operative intervention in patients with cardiovascular disease. Anaesthesia 2005;60:1106-14.

4 Karthikeyan G, Bhargava B. Managing patients undergoing non-cardiac surgery: need to shift emphasis from risk stratification to risk modification. Heart 2006;92:17-20.

5 Biccard BM, Sear JW, Foex P. The pharmaco-economics of peri-operative statin therapy. Anaesthesia 2005;60:1059-63.

6 Cholesterol Treatment Trialists' Collaborators. Efficacy and safety of cholesterollowering treatment: prospective meta-analysis of data from 90056 participants in 14 lowering treatment. prospective meta-analysis of dat

7 Landesberg G. The pathophysiology of perioperative myocardial infarction: facts and perspectives.J Cardiothorac Vasc Anesth 2003;17:90-100.

8 Downs SH, Black N. The feasibility of creating a checklist for the assessment of the methodological quality both of randomised and non-randomised studies of healthcare interventions.J Epidemiol Community Health 1998;52:377-84.

9 Jadad AR, Moore RA, Carroll D, Jenkinson C, Reynolds DJ, Gavaghan DJ, et al. Assessing the quality of reports of randomized clinical trials: Is blinding necessary? Contro Clin Trials 1996;17:1-12.

10 Egger M, Davey Smith G, Schneider M, Minder C. Bias in meta-analysis detected by a simple, graphical test . BMJ 1997;315:629-34.

11 Christenson JT. Preoperative lipid control with simvastatin reduces the risk for graft failure already 1 year after myocardial revascularization. Cardiovasc Surg 2001;9:33-43. 12 Kertai MD, Boersma E, Westerhout CM, van Domburg R, Klein J, Bax JJ, et al. Association between long-term statin use and mortality after successful abdominal aortic neurysm surgery. Am J Med 2004;116:96-103.

13 Ali IS, Buth KJ. Preoperative statin use and in-hospital outcomes following hear surgery in patients with unstable angina. Eur J Cardiothorac Surg 2005;27:1051-6.

14 Knatterud GL, Rosenberg Y, Campeau L, Geller NL, Hunninghake DB, Forman SA, et al. Long-term effects on clinical outcomes of aggressive lowering of low-density lipoprotein cholesterol levels and low-dose anticoagulation in the post coronary artery bypass graft trial. Post CABG Investigators. Circulation 2000;102:157-65.

15 Schwartz GG, Olsson AG, Ezekowitz MD, Ganz P, Oliver MF, Waters D, et al. Effects of atorvastatin on early recurrent ischemic events in acute coronary syndromes: the MIRACL study: a randomized controlled trial. JAMA 2001;285:1711-8.
16 De Lemos JA, Blazing MA, Wiviott SD, Lewis EF, Fox KA, White HD, et al. Early intensive vs a delayed conservative simvastatin strategy in patients with acute coronary syndromes: phase $\mathrm{Z}$ of the A to Z Trial.JAMA 2004;292:1307-16.

17 Schouten O, Poldermans D. Statins in the prevention of perioperative cardiovascular complications. Curr Opin Anaesthesiol 2005;18:51-5.

18 Herrington DM. Hormone replacement therapy and heart disease. Replacing dogma with data. Circulation 2003;107:2-4.

19 Spencer FA, Fonarow GC, Frederick PD, Wright RS, Every N, Goldberg RJ, et al. Early withdrawal of statin therapy in patients with non-ST-segment elevation myocardial infarction: national registry of myocardial infarction. Arch Intern Med 2004;164:2162-8.

20 Spencer FA, Allegrone J, Goldberg RJ, Gore JM, Fox KA, Granger CB, et al. Association of statin therapy with outcomes of acute coronary syndromes: the GRACE study. An Intern Med 2004;140:857-66.

(Accepted 22 September 2006)

doi 10.1136/bmj.39006.531146.BE

Division of General Internal Medicine, University of Alberta, 8440112 Street, Edmonton, AB, Canada T6G 2R7

Anmol S Kapoor postgraduate trainee

Finlay A McAlister associate professor

Department of Cardiovascular Surgery, University of Alberta

Hussein Kanji postgraduate trainee

John W Scott Health Sciences Library, University of Alberta

Jeanette Buckingham medical librarian

Department of Medicine, Clinical Epidemiology, and Biostatistics, McMaster

University, Hamilton, Canada

P J Devereaux assistant professor

Correspondence to: F A McAlister finlay.mcalister@ualberta.ca 